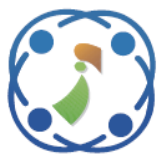

\title{
Football Game Based Optimization: an Application to Solve Energy Commitment Problem
}

\author{
Mohammad Dehghani ${ }^{1 *}$ \\ Mohammad Mardaneh ${ }^{1}$ \\ O. P. Malik ${ }^{3} \quad$ Vijay Kumar $^{4}$ \\ Josep M. Guerrero \\ ${ }^{I}$ Dept. of Electrical and Electronics Engineering, Shiraz University of Technology, Shiraz \\ ${ }^{2}$ Center for Research on Microgrids (CROM), Department of Energy Technology, \\ Aalborg University, Aalborg, Denmark \\ ${ }^{3}$ Departmentof Electrical Engineering, University of Calgary, Calgary, Alberta, Canada \\ ${ }^{4}$ Computer Science and Engineering Department, National Institute of Technology, Hamirpur, India \\ * Corresponding author's Email: adanbax@gmail.com
}

\begin{abstract}
Heuristic optimization algorithms are widely used to solve problems in different fields of science. In this paper, a new game based optimization method called football game based optimization (FGBO) is presented which simulates the game of football. The population of FGBO are clubs and the variables of the problem are the players belonging to the clubs. FGBO has four phases: a) league holding, b) player transfer, c) practice, and d) promotion and relegation. The power of FGBO in solving optimization problems has been investigated on several benchmark test functions. The result of FGBO and other algorithm are obtained from implantation of these algorithms on unimodal, multimodal, and fixed-dimension multimodal benchmark test functions. Eight optimization algorithms called Genetic Algorithm (GA), Particle Swarm Optimization (PSO), Teaching Learning Based Optimization (TLBO), Grey Wolf Optimizer (GWO), Grasshopper Optimization Algorithm (GOA), Emperor Penguin Optimizer (EPO), Shell Game Optimization (SGO), and Hide Objects Game Optimization (HOGO) have been used to compare these results. The proposed FGBO algorithm is also used to solve the energy commitment (EC) problem. Based on the simulation studies and obtained results, FGBO has a higher efficiency than a number of other algorithms. The results and data obtained from applying FGBO and other mentioned algorithms on unimodal test functions, multimodal test functions, and energy commitment problem show that FGBO is able to provide better results in comparison with other well-known optimization algorithms.
\end{abstract}

Keywords: Energy commitment, Football, Football game based optimization, Game, Game based algorithms, Optimization.

\section{Introduction}

\subsection{Motivation}

In recent decades, heuristic optimization algorithms have been widely used in applied science field. The power of these algorithms to solve optimization problems is undeniable. Such algorithms have demonstrated their high ability in many fields such as energy [1], protection [2], electrical engineering [3-7], and energy carriers [8,9] to achieve the optimal solution.

\subsection{Contribution}

In recent years, the use of optimization algorithms has become very common. Numerous algorithms have been proposed by scientists, most of which are based on the laws of physics, evolution, and swarm activities. However, mathematical modeling of phenomena in which the process of evolution and progress lies, can provide new optimizers. Game is a social activity in which participants, called game players, try to win according to the rules of the game. Therefore, simulating this process of achieving 
victory considering the rules of the game, can lead to the design of new optimization algorithms.

A new game based optimization method, called football game based optimization (FGBO) that simulates the game of football, is proposed in this paper. The population of FGBO is clubs and the variables of the problem are the players in the clubs. FGBO has three phases: a) league holding, b) player transfer, c) practice, and d) promotion and relegation.

Many of the scientists' proposed optimization algorithms face two challenges: complexity of the equations and setting multiple control parameters. However, the simplicity of the equations and the lack of control parameters, as well as the implementation of the proposed algorithm of the authors of this paper, are important features of FGBO. Therefore, FGBO can be easily applied to any optimization problem. The performance of FGBO has been compared to eight well-known optimization techniques considering twenty-three linear and nonlinear benchmark test functions. Moreover, FGBO has been tested on an engineering optimization problem named energy commitment to validate its effectiveness.

\subsection{Paper organization}

A background of the optimization algorithms is given in Section 2. The proposed football game based optimization algorithm is presented in Section 3. Simulation results are described in Section 4 and conclusions are drawn in Section 5.

\section{Background}

Each optimization problem is mathematically modeled in three parts, including objective functions, variables, and constraints. The goal in optimization is to minimize or maximize the objective functions considering constraints. There are several ways to solve an optimization problem. One of these methods is optimization algorithms, which always have a high ability to solve optimization problems. Optimization algorithms, without the need for a derivation process, provide a quasi-optimal answer by randomly searching in the defined space of the variables. The term quasi-optimization is used because optimization algorithms randomly check the search space may not be able to achieve the optimal answer. However, the quasi-optimal answer is an answer close to the optimal answer. Therefore, the advantage of one algorithm over another algorithm is in providing a quasi-optimal answer that is very close to the optimal answer. Due to this, several optimization algorithms have been developed by researchers to obtain more appropriate answers. In the theoretical mode, the difference between optimization algorithms is in main design idea, the complexity of the equations, and the existence of control parameters and their adjustment. However, in order to compare optimization algorithms more deeply, these algorithms should be implemented on optimization problems and analyzed based on the obtained results.

Heuristic optimization algorithms can be divided into four categories. These categories are: physicsbased algorithm, swarm-based algorithm, gamebased algorithm, and evolution-based algorithms.

\subsection{Physics-based algorithm}

Physics-based algorithms are based on the simulation of the rules of physics. Some of this kind of algorithms are: Black Hole (BH) [10] based on black hole phenomenon, Gravitation Search Algorithm (GSA) [11] based on gravitational gravity force, Spring Search Algorithm (SSA) [12, 13] inspired by Hooke's law, Charged System Search (CSS) [14] based on some principles from physics and mechanics where each agent is a charged particle, Curved Space Optimization (CSO) [15] based on transformation of a random search space into a new search space based on the concepts of space-time curvature in general relativity theory, Galaxy-based Search Algorithm (GbSA) [16] based on spiral arm of spiral galaxies, Ray Optimization (RO) [17] algorithm based on the Snell's light refraction law, Small World Optimization Algorithm (SWOA) [18] based on mechanism of small-world phenomenon, and Artificial Chemical Reaction Optimization Algorithm (ACROA) [19] based on chemical reactions processes.

\subsection{Swarm-based algorithm}

Swarm-based algorithms are inspired from the natural processes of plants, foraging behavior of insects and social behavior of animals. Some of the known optimization algorithms belonging to this category are: Donkey Theorem Optimization (DTO) [20], Particle Swarm Optimization (PSO) [21], Artificial Bee Colony (ABC) [22], Ant Colony Optimization (ACO) [23], Bat-inspired Algorithm (BA) [24], Emperor Penguin Optimizer (EPO) [25], Spotted Hyena Optimizer (SHO) [26], Gray Wolf Optimizer (GWO) [27], Cuckoo Search (CS) [28], Following Optimization Algorithm (FOA) [29], Rat Swarm Optimizer (RSO) [30], Group Optimization (GO) [31], and Grasshopper Optimization Algorithm (GOA) [32]. 


\subsection{Game-based algorithm}

Some optimization algorithms such as Orientation Search Algorithm (OSA) [33] which simulates rules of orientation game or Dice Game Optimizer (DGO) [34] that simulates the rules of an old game named dice game are the members of Game-based algorithms which are based on simulation of different games. Shell Game Optimization (SGO) [35], Hide Objects Game Optimization (HOGO) [36], and Darts Game Optimizer (DGO) [37] are part of this group of algorithms.

\subsection{Evolution-based algorithms}

Evolutionary algorithms have involved evolution of a population in order to create new generations of genetically superior individuals [38]. Differential Evolution (DE) [39], Genetic Algorithm (GA) [40], Genetic Programming (GP) [41], Evolution Strategy (ES) [42], and Biogeography-based Optimizer (BBO) [43] are part of this group of algorithms.

\section{Football game based optimization (FGBO)}

In this paper, an optimization method named Football game based optimization, which simulates the football game, is presented. FGBO is simulated in four phases: a) league holding, b) player transfer, c) practice, and d) promotion and relegation.

Population of FGBO are clubs that are specified by Eq. (1):

$$
C_{i}=\left[c_{i}^{1}, \ldots, c_{i}^{d}, \ldots, c_{i}^{m}\right]
$$

Here, $C_{i}$ is $i$-th club, $c_{i}^{d}$ is $d$-th member of $i$-th club, and $m$ is the number of players.

The power of clubs is evaluated by Eq. (2) and the best club is specified by Eq. (3):

$$
\begin{gathered}
P_{i}=\frac{f i t_{i}-\max (f i t)}{\sum_{j=1}^{N}\left[\text { fit }_{j}-\max (f i t)\right]} \\
C_{\text {best }}=\text { the position of } \min (f i t)
\end{gathered}
$$

Here, $P_{i}$ is the power of the $i$-th club, $f i t_{i}$ is the fitness function of $i$-th agent, and $N$ is the number of clubs.

\subsection{League holding}

There are three modes for each club in each game. In fact, the outcome of a match for a club may be a win, a loss or a draw that are simulated by Eqs. (4) to (8).

$$
\begin{gathered}
z_{i}^{\text {win }}=0.95 \frac{P_{i}}{P_{i}+P_{j}} \\
z_{i}^{\text {loss }}=0.95 \frac{P_{j}}{P_{i}+P_{j}} \\
z_{i}^{\text {draw }}=1-\left(z_{i}^{\text {win }}+z_{i}^{\text {loss }}\right)=0.05 \\
S_{i, j}= \begin{cases}3, & 0<r<z_{i}^{\text {win }} \\
1, & z_{i}^{\text {win }}<r<1-z_{i}^{\text {loss }} \\
0, & \text { else }\end{cases} \\
S_{j, i}= \begin{cases}0, & 0<r<z_{i}^{\text {win }} \\
1, & z_{i}^{\text {win }}<r<1-z_{i}^{\text {loss }} \\
3, & \text { else }\end{cases}
\end{gathered}
$$

Here, $z_{i}^{\text {win }}, z_{i}^{\text {loss }}$, and $z_{i}^{\text {draw }}$ are the probability of winning, losing, and drawing of the $i$-th club, and $S_{i, j}$ is the score of the $i$-th club in face of $j$-th club.

\subsection{Player transfer}

In this phase, each club employs a few new players based on their points. This phase is simulated by Eq. (9) to by Eq. (11).

$$
\begin{gathered}
N_{p t_{i}}=\text { round }\left[0.5 \times m\left(1-\frac{t}{T}\right) \times\left(\frac{\text { rank }_{i}-1}{N-1}\right)\right](9) \\
D_{p t_{i}}=\operatorname{randperm}\left(m, N_{p t_{i}}\right) \\
C_{i}\left(D_{p t_{i}}\right)=C_{\text {best }}\left(D_{p t_{i}}\right)
\end{gathered}
$$

Here, $N_{p t_{i}}$ is the number of transitional players to $i$-th club, $t$ is the number of iteration, $T$ is the maximum number of iterations, $\operatorname{rank}_{i}$ is the position of $i$-th club in league table, and $D_{p t_{i}}$ is the position of transferred players to $i$-th club.

\subsection{Practice}

In this phase, each club is trying to improve its situation with practice. In fact, a new agent (club) is created in the neighborhood of each agent (club). If the new agent is better, the status of the club will be updated. This phase is simulated by Eq. (12) to Eq. (14).

$$
\begin{gathered}
C_{i}^{\prime}=\left(1-I_{P_{i}}\right) C_{i}+I_{P} C_{\text {best }} \\
I_{P_{i}}=0.2 \frac{\text { rank }_{i}}{N}\left(1-\frac{t}{T}\right)
\end{gathered}
$$




$$
C_{i}=\left\{\begin{array}{l}
C_{i}, \text { fit }_{i}<f i t_{i}^{\prime} \\
C_{i}^{\prime}, \text { else }
\end{array}\right.
$$

Here, $C_{i}^{\prime}$ is the status and $f i t_{i}^{\prime}$ is the fitness function of $i$-th club after practice. In addition, $I_{P_{i}}$ is the index of prace.

\subsection{Promotion and Relegation}

In this phase, some poor clubs are eliminated from the league and some new qualified clubs substitute them in the league. This process is simulated by (15) to (16).

$$
\begin{gathered}
N_{p r}=0.1 \times N \times\left(1-\frac{t}{T}\right) \\
C_{i}=C_{i}+2 \times \text { rand } \times\left(C_{\text {best }}-C_{i}\right) \\
\& i=N-N_{p r}+1: N
\end{gathered}
$$
clubs.

Here, $N_{p r}$ is the number of promoted or relegated

The various steps of FGBO are as follows:

Start

a) Set up the system.

b) The initial status of the clubs.

c) Evaluation of clubs.

d) $C_{\text {best }}$ and power update.

e) Phase a: league holding (4) to (8).

f) Phase b: player transfer (9) to (11).

g) Phase c: practice (12) to (14).

h) Phase d: promotion and relegation (15) to (16).

i) Update of the status of the clubs.

j) As long as the stop condition is not satisfied, End repeat steps $\mathrm{c}$ to $\mathrm{i}$.

\section{Simulation}

In this section the performance of the FGBO is evaluated by applying the standard benchmark test functions adapted from [44]. In order to demonstrate the effectiveness of the FGBO, it is compared with eight well-known optimization algorithms (GA, PSO, TLBO, GWO, GOA, EPO, SGO, and HOGO) on unimodal, multimodal, and fixed-dimension multimodal benchmark test functions.

The experimentation has been done on MATLAB R2014a version in the environment of Microsoft Windows 7 using 64 bit Core i-7 processor with 2.40 $\mathrm{GHz}$ and $8 \mathrm{~GB}$ main memory. The average and standard deviations of the best optimal solution are mentioned in Tables 1-3.

\subsection{Unimodal test functions with high dimension}

The results of evaluation of FGBO and other algorithms on functions $F_{1}$ to $F_{7}$ given in Table 1 show that FGBO has better performance than other algorithms in all Unimodal test functions with high dimension. In Table 1, Avg and std stand for average value and standard deviation of obtained results.

\subsection{Multimodal test functions with high dimension}

In multimodal test functions, $F_{8}$ to $F_{13}$, the number of local solutions increases exponentially with increasing function dimensions. Therefore, it is hardly possible to achieve the global minimum in these types of functions. Instead, reaching the nearest solution to global minimum indicates the high power of the algorithm in passing the wrong local solution. The results of evaluating functions $F_{8}$ to $F_{13}$ are shown in Table 2. In all of these functions, FGBO shows better performance.

\subsection{Multimodal test functions with low dimension}

Functions $\mathrm{F}_{14}$ to $\mathrm{F}_{23}$ have a low number of dimensions and they also have few local minimums. Results of the 20-time implementation of FGBO and other algorithms are presented in Table 3. These results show the proper performance of FGBO in optimizing these types of functions.

\section{4 theoretical explanation and discussion}

Two important indicators in comparing the ability of optimization algorithms in solving optimization problems are exploration and exploitation power. The exploration index examines the ability of an optimization algorithm to search properly and comprehensively in the search space of the problem. Therefore, an optimization algorithm that searches the search space more accurately has higher exploration power. This prevents the algorithm from getting stuck in local optimizations points. The exploitation index is the study of the ability of an optimization algorithm to provide an appropriate quasi-optimal response. Therefore, an algorithm that provides a quasi-optimal answer closer to the optimal answer has a higher exploitation power. The important principle is to maintain a balance between these two indicators during the repetition of the algorithm. Thus, in the initial iterations, the exploration power should be a priority so that the search space is searched accurately. Then, with the repetition of the algorithm repetitions, the exploration power is reduced and the exploitation power is given priority so that the quasi-optimal answer is presented. 
Based on this important principle, an algorithm that can accurately search the problem search space is able to provide a better quasi-optimal answer. The proposed FGBO algorithm, by maintaining a proper balance between the two exploration and exploitation indicators, has been able to provide appropriate quasi-optimal answers for all three types of unimodal, multimodal, and fixed-dimension multimodal objective functions.

The results presented in Tables 1 to 3 show that the proposed FGBO algorithm has a clear advantage over all other eight algorithms in all twenty-three objective functions. Functions $F_{1}$ to $F_{7}$ have been proposed to analyze the exploitation ability of optimization algorithms. According to Table 1, FGBO has higher exploitation ability than the other eight algorithms.

Objective functions $\mathrm{F}_{8}$ to $\mathrm{F}_{23}$ have also been proposed to analyze the exploration capability of optimization algorithms. Based on Tables 2 and 3, the proposed FGBO algorithm has a higher exploration capability than the other eight algorithms.

\section{FGBO for energy commitment solving}

In this section, FGBO performance is assessed on the energy commitment (EC) problem. Energy consumption is an important indicator in determining the quality of life in any society. Therefore, the proper use of energy resources and carriers is an important principle in energy operation. The goal of $\mathrm{EC}$ is to determine the most appropriate way to use energy carriers to meet energy demand by taking into account technical and economic constraints. Therefore, EC is a constrained optimization problem that can be solved using optimization algorithms [9].

Unit commitment (UC) is one of the important studies of electricity networks. The purpose of UC is to determine the most appropriate composition and production of power plant for each hour of the study period to supply demand considering technical and economic constraints. In UC, the operation of the power carrier is optimized independently. However, the interdependencies between different energy networks make integrated energy network studies very important. The demand of energy consumers at different levels consists of different types of energy carriers such as gas, electricity, gasoline, etc., which must be optimally supplied. The study of EC optimizes this operation. In EC, the objective function is to reduce the operating costs of different energy carriers in order to meet energy demand, decision-making variables are the amount of energy carriers, and the most important constraint is the balance of energy supply and demand.

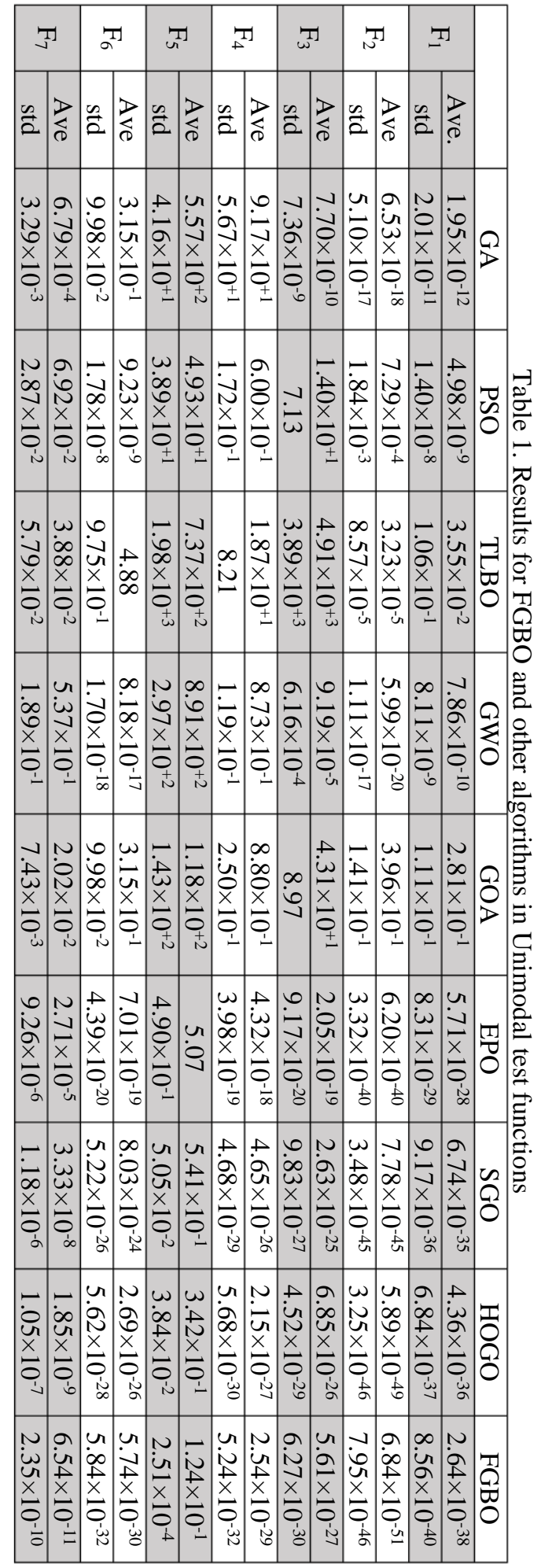

International Journal of Intelligent Engineering and Systems, Vol.13, No.5, 2020

DOI: $10.22266 /$ ijies2020.1031.45 


\begin{tabular}{|c|c|c|c|c|c|c|c|c|c|c|c|c|}
\hline \multicolumn{2}{|c|}{$\mathbb{\omega}_{\omega}^{T}$} & \multicolumn{2}{|c|}{$\frac{T}{N}$} & \multicolumn{2}{|c|}{$\stackrel{T}{I}$} & \multicolumn{2}{|c|}{$\frac{T}{0}$} & \multicolumn{2}{|c|}{$\sigma^{T}$} & \multicolumn{2}{|c|}{$\infty_{\infty}^{T}$} & \\
\hline$\tilde{a}$ & 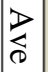 & $\tilde{a}$ & $\begin{array}{l}\vec{D} \\
\grave{d}\end{array}$ & $\underline{a}$ & 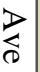 & $\stackrel{n}{\tilde{a}}$ & 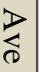 & $\underline{a}$ & $\begin{array}{l}\vec{x} \\
< \\
\Delta\end{array}$ & $\underline{a}$ & 衤 & \\
\hline 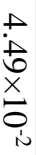 & 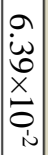 & 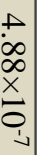 & $\begin{array}{l}0 \\
\vec{a} \\
\dot{x} \\
0 \\
0 \\
\dot{\infty}\end{array}$ & 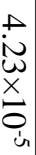 & $\begin{array}{l}w \\
w \\
w \\
x \\
\underline{x} \\
\vdots \\
\alpha\end{array}$ & $\begin{array}{l}\overline{\bar{x}} \\
\frac{\bar{x}}{0} \\
\frac{1}{0}\end{array}$ & 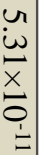 & 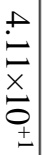 & 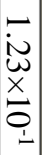 & 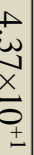 & 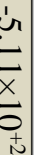 & $\Omega$ \\
\hline 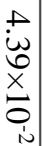 & 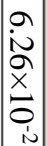 & 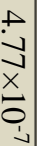 & $\begin{array}{l}\infty \\
\dot{b} \\
\omega \\
x \\
0 \\
0 \\
\infty\end{array}$ & 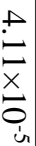 & 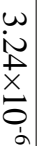 & $\begin{array}{l}\dot{\infty} \\
\stackrel{\infty}{x} \\
\dot{0}\end{array}$ & 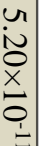 & $\begin{array}{l}\stackrel{+}{0} \\
\frac{\hat{x}}{0} \\
0\end{array}$ & 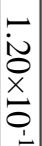 & & 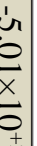 & $\widetilde{\sigma}$ \\
\hline
\end{tabular}

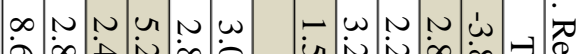

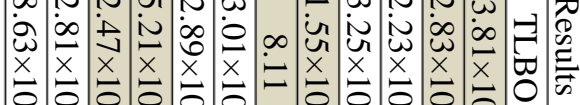

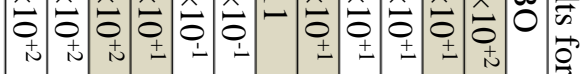

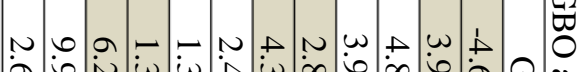

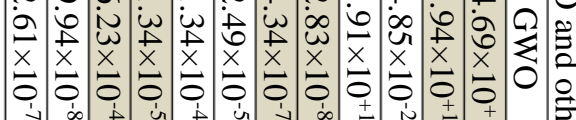

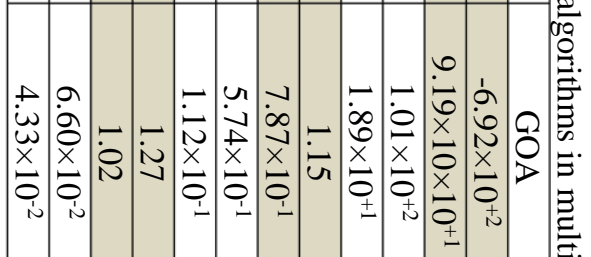

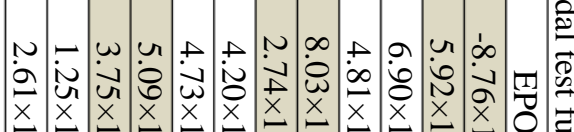

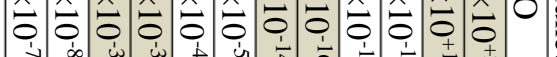

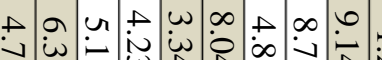

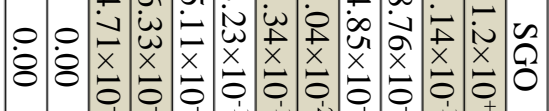

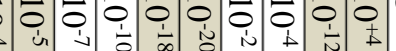

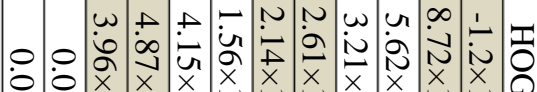

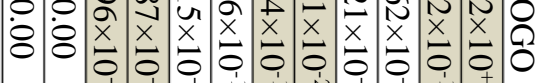

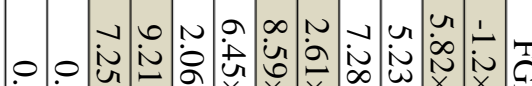

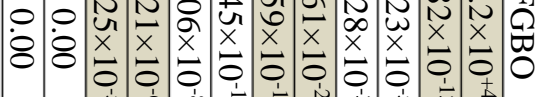

\begin{tabular}{|c|c|c|c|c|c|c|c|c|c|c|c|c|c|c|c|c|c|c|c|}
\hline స్త & Ty & N & & 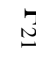 & ?] & సิ & & 7 & a & $\frac{T}{\infty}$ & & $\bar{J}$ & & ${ }^{T}$ & & $\frac{T}{n}$ & & $\underset{F}{T}$ & \\
\hline$\stackrel{n}{a}$ & 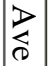 & $\stackrel{\tilde{a}}{\tilde{D}}$ & 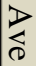 & $\vec{z}$ & 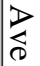 & $\tilde{a}$ & 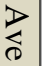 & 竞 & 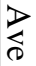 & $\stackrel{n}{\tilde{D}}$ & 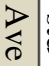 & $\underline{\tilde{z}}$ & 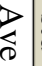 & & & $\overrightarrow{2}$ & & $\frac{2}{2} \frac{2}{\delta}$ & \\
\hline 芯 & 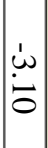 & 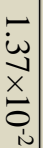 & لَّ & $\overbrace{+}^{N}$ & r & $\begin{array}{l}+ \\
\dot{\omega} \\
\tilde{x} \\
\underline{x} \\
\dot{0}\end{array}$ & 'ِ & $\begin{array}{l}x^{\prime} \\
\underline{x}\end{array}$ & سِ & $\begin{array}{l}a \\
\dot{\omega} \\
\omega \\
x \\
0 \\
0 \\
\dot{u}\end{array}$ & w & 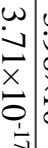 & $\begin{array}{l}\omega \\
0 \\
\infty \\
x \\
0 \\
0 \\
\end{array}$ & 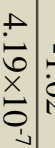 & $\frac{1}{2}$ & 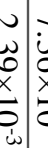 & 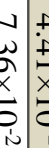 & 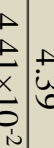 & 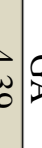 \\
\hline
\end{tabular}

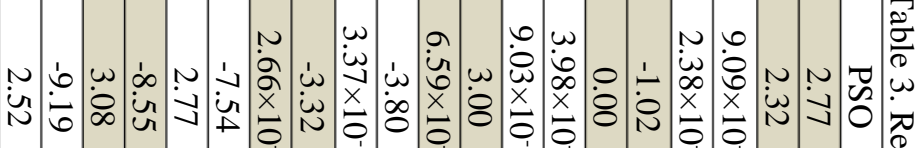

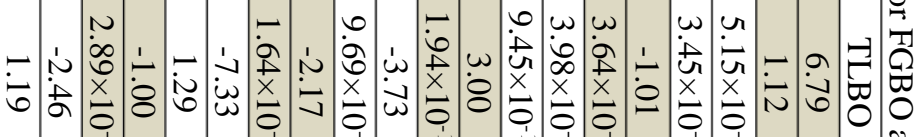

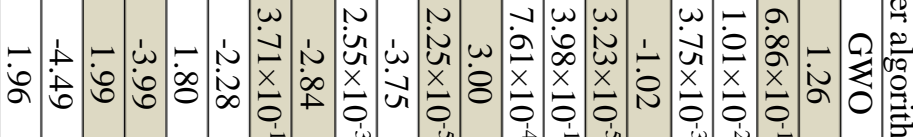

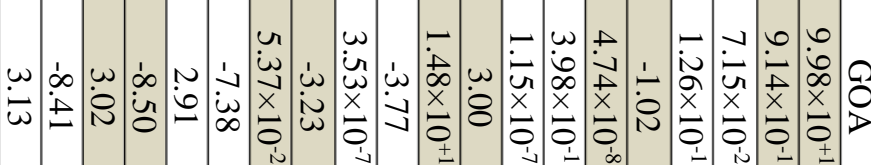

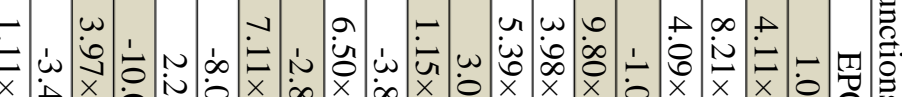

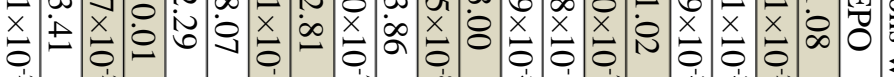

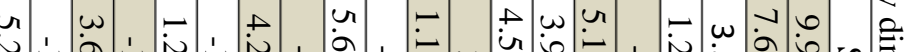
مَ)

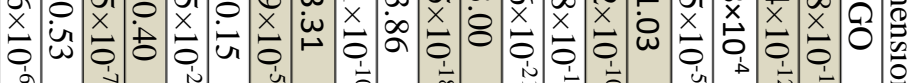

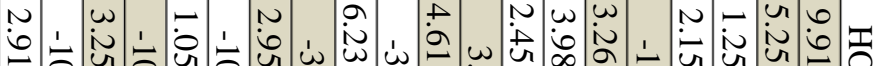

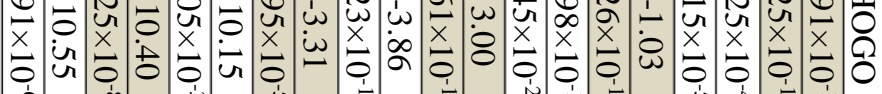

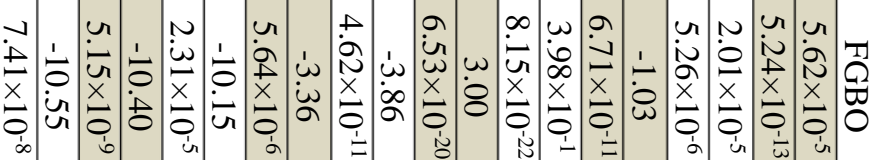


The mathematical model of energy commitment, including the objective function and constraint, is as follows:

$$
\begin{gathered}
F_{\text {objective }} \\
=\min \left\{\sum _ { t = 1 } ^ { T } \left[\sum_{i=1}^{N_{c}} \text { carrier }_{i}^{t} \times \text { price }_{i}+\right.\right. \\
\left.\left.\quad \sum_{i=1}^{N_{g}} S C_{i}^{t}+\sum_{i=1}^{N_{g}} C_{i} u_{i}^{t}\right]\right\} \\
S C_{i}^{t}=\left\{\begin{array}{c}
S C_{i}, u_{i}^{t}>u_{i}^{t-1} \\
0, \text { else }
\end{array}\right. \\
P_{g_{i}}^{\text {min }} \leq P_{g_{i}} \leq P_{g_{i}}^{\max } \\
\sum_{i=1}^{N_{g}} P_{g_{i}}^{t}=\text { load }^{t}
\end{gathered}
$$

Here $T$ is the duration of study period, $N_{c}$ is the number of energy carriers, $N_{g}$ is the number of units, $S C$ is the startup cost of units, $C$ is the constant cost of units, $u$ is the status of on or off for each units, $P_{g}$ is the value of production of each units, $P_{g}^{\min }$ and $P_{g}^{\max }$ are the minimum and maximum of production of each units respectively, and load is the demand in network.

The results of the implementation of FGBO and the eight mentioned algorithms are presented in Table 4. The proposed FGBO has provided the most optimal answer in this comparison at a cost of 2.1153E+07 Dollars.

The results presented in Table 4 show that the proposed FGBO algorithm is also effective in solving real-world optimization problems. FGBO has been able to provide the best quasi-optimal response than the other eight algorithms by carefully searching the search space and avoiding local optimal answers and

\begin{tabular}{|c|c|c|}
\hline \multicolumn{3}{|c|}{ problem } \\
\hline & Ave (Dollar) & Std (Dollar) \\
\hline GA & $8.5146 \times 10^{8}$ & $2.6145 \times 10^{6}$ \\
\hline PSO & $5.2158 \times 10^{8}$ & $1.2485 \times 10^{6}$ \\
\hline TLBO & $6.7624 \times 10^{7}$ & $5.2176 \times 10^{4}$ \\
\hline GWO & $3.2648 \times 10^{7}$ & $7.5423 \times 10^{3}$ \\
\hline GOA & $2.7592 \times 10^{7}$ & $8.6427 \times 10^{2}$ \\
\hline EPO & $2.4257 \times 10^{7}$ & $6.5654 \times 10^{2}$ \\
\hline SGO & $2.1739 \times 10^{7}$ & $2.7865 \times 10^{2}$ \\
\hline HOGO & $2.2365 \times 10^{7}$ & $1.4552 \times 10^{2}$ \\
\hline FGBO & $2.1153 \times 10^{7}$ & 8.6123 \\
\hline
\end{tabular}
maintaining a balance between exploration and exploitation indicators. This illustrates the obvious superiority of the FGBO over the other eight algorithms in solving the EC problem.

Table 4. Results for FGBO and other algorithms in EC

\section{Conclusions}

Heuristic optimization algorithms are increasingly being used to solve optimization problems in various applications. These algorithms can be divided into four categories: physics-based algorithm, swarm-based algorithm, game-based algorithm, and evolutionary algorithms. In this regard, a new game-based algorithm called Football game based optimization (FGBO) based on the football game is presented in this paper. FGBO is based on rules of football game which clubs and players try to achieve to victory and championship. In FGBO, football game is simulated in four phase: a) league holding, b) player transfer, c) practice, and d) promotion and relegation. With the mathematical modeling of these four phases, FGBO has been designed as an optimizer that can be used to solve optimization problems in various sciences.

The performance of FGBO is evaluated on a set of standard benchmark test functions, which is categorized to three groups named unimodal, multimodal, and fixed-dimension multimodal benchmark test functions. The results show that FGBO provides very competitive results as compared with other well-known optimization algorithms such as GA, PSO, TLBO, GWO, GOA, EPO, SGO, and HOGO.

Moreover, the proposed FGBO algorithm is used to solve the energy commitment (EC) problem. EC is a new challenge in power system studies that it can be solved with the optimization algorithms. The proposed algorithm performs well in EC problem solving than to existing algorithms.

\section{Conflicts of Interest}

The authors declare no conflict of interest.

\section{Author Contributions}

Conceptualization, M. Dehghani, M. Mardaneh, and J. M. Guerrero.; methodology, M. Dehghani and M. Mardaneh.; software, M. Dehghani and V. Kumar.; validation, J. M. Guerrero, V. Kumar, and O.P. Malik.; formal analysis, V. Kumar and O.P. Malik.; investigation, M. Dehgani and M. Mardaneh.; resources, J. M. Guerrero.; data curation, V. Kumar and O.P. Malik; writing - original draft preparation, M. Dehghani and M. Mardaneh.; writing - review and editing, V. Kumar and O.P. Malik.; visualization, M. Dehghani.; supervision, M. Dehghani.; project administration, M. Dehghani and M. Mardaneh; funding acquisition, J. M. Guerrero. 


\section{Acknowledgments}

This work was supported by VILLUM FONDEN under the VILLUM Investigator Grant (no. 25920): Center for Research on Microgrids (CROM); www.crom.et.aau.dk

\section{References}

[1] M. Dehghani, Z. Montazeri, and O. P. Malik, "Energy Commitment: A Planning of Energy Carrier Based on Energy Consumption", Electrical Engineering \& Electromechanics, No. 4, pp. 69-72, 2019.

[2] A. Ehsanifar, M. Dehghani, and M. Allahbakhshi, "Calculating The Leakage Inductance for Transformer Inter-Turn Fault Detection Using Finite Element Method", In: Proc. of Iranian Conf. on Electrical Engineering (ICEE), Tehran, Iran, pp. 1372-1377, 2017.

[3] M. Dehghani, Z. Montazeri, and O. P. Malik, "Optimal Sizing and Placement of Capacitor Banks and Distributed Generation in Distribution Systems Using Spring Search Algorithm", International Journal of Emerging Electric Power Systems, Vol. 21, No. 1, 20190217, 2020.

[4] M. Dehghani, Z. Montazeri, O. P. Malik, K. AlHaddad, J. M. Guerrero, and G. Dhiman, "A New Methodology Called Dice Game Optimizer for Capacitor Placement in Distribution Systems", Electrical Engineering \& Electromechanics, No. 1, pp. 61-64, 2020.

[5] S. Dehbozorgi, A. Ehsanifar, Z. Montazeri, M. Dehghani, and A. Seifi, "Line Loss Reduction and Voltage Profile Improvement in Radial Distribution Networks Using Battery Energy Storage System", In: Proc. of IEEE 4th International Conf. on Knowledge-Based Engineering and Innovation (KBEI), Tehran, Iran, pp. 0215-0219, 2017.

[6] Z. Montazeri and T. Niknam, "Optimal Utilization of Electrical Energy from Power Plants Based on Final Energy Consumption Using Gravitational Search Algorithm", Electrical Engineering \& Electromechanics, No. 4, pp. 70-73, 2018.

[7] M. Dehghani, M. Mardaneh, Z. Montazeri, A. Ehsanifar, M. Ebadi, and O. Grechko, "Spring Search Algorithm for Simultaneous Placement of Distributed Generation and Capacitors", Electrical Engineering \& Electromechanics, No. 6, pp. 68-73, 2018.

[8] M. Dehghani, Z. Montazeri, A. Ehsanifar, A. Seifi, M. Ebadi, and O. Grechko, "Planning of Energy Carriers Based on Final Energy
Consumption Using Dynamic Programming and Particle Swarm Optimization", Electrical Engineering \& Electromechanics, No. 5, pp. 6271, 2018.

[9] Z. Montazeri and T. Niknam, "Energy Carriers Management Based on Energy Consumption", In: Proc. of IEEE 4th International Conf. on Knowledge-Based Engineering and Innovation (KBEI), Tehran, Iran, pp. 0539-0543, 2017.

[10] A. Hatamlou, "Black Hole: A New Heuristic Optimization Approach for Data Clustering", Information Sciences, Vol. 222, pp. 175-184, 2013.

[11] E. Rashedi, H. Nezamabadi-Pour, and S. Saryazdi, "GSA: A Gravitational Search Algorithm", Information Sciences, Vol. 179, pp. 2232-2248, 2009.

[12] M. Dehghani, Z. Montazeri, A. Dehghani, and A. Seifi, "Spring Search Algorithm: A New MetaHeuristic Optimization Algorithm Inspired by Hooke's Law", In: Proc. of IEEE 4th International Conf. on Knowledge-Based Engineering and Innovation (KBEI), Tehran, Iran, pp. 0210-0214, 2017.

[13] M. Dehghani, Z. Montazeri, A. Dehghani, N. Nouri, and A. Seifi, "BSSA: Binary Spring Search Algorithm", In: Proc. of IEEE 4th International Conf. on Knowledge-Based Engineering and Innovation (KBEI), Tehran, Iran, pp. 0220-0224, 2017.

[14] A. Kaveh and S. Talatahari, "A Novel Heuristic Optimization Method: Charged System Search", Acta Mechanica, Vol. 213, pp. 267-289, 2010.

[15] F. F. Moghaddam, R. F. Moghaddam, and M. Cheriet, "Curved Space Optimization: A Random Search Based on General Relativity Theory", arXiv preprint arXiv: 1208.2214, 2012.

[16] H. Shah-Hosseini, "Principal Components Analysis by the Galaxy-Based Search Algorithm: A Novel Metaheuristic for Continuous Optimisation", International Journal of Computational Science and Engineering, Vol. 6, pp. 132-140, 2011.

[17] A. Kaveh and M. Khayatazad, "A New MetaHeuristic Method: Ray Optimization", Computers \& Structures, Vol. 112, pp. 283-294, 2012.

[18] H. Du, X. Wu, and J. Zhuang, "Small-World Optimization Algorithm for Function Optimization", In: Proc. of International Conf. on Natural Computation, pp.264-273, 2006.

[19] B. Alatas, "ACROA: Artificial Chemical Reaction Optimization Algorithm for Global Optimization", Expert Systems with Applications, Vol. 38, pp. 13170-13180, 2011. 
[20] M. Dehghani, M. Mardaneh, O. P. Malik, and S. M. NouraeiPour, "DTO: Donkey Theorem Optimization", In: Proc. of 27th Iranian Conf. on Electrical Engineering (ICEE), Yazd, Iran, pp. 1855-1859, 2019.

[21] J. C. Bansal, "Particle Swarm Optimization", In: Evolutionary and Swarm Intelligence Algorithms, ed: Springer, pp. 11-23, 2019.

[22] D. Karaboga and B. Basturk, "Artificial Bee Colony (ABC) Optimization Algorithm for Solving Constrained Optimization Problems", In: Proc. of International fuzzy systems association world congress, pp. 789-798, 2007.

[23] M. Dorigo and T. Stützle, "Ant Colony Optimization: Overview and Recent Advances", In: Handbook of Metaheuristics, ed: Springer, pp. 311-351, 2019.

[24] X.-S. Yang, "A New Metaheuristic Bat-Inspired Algorithm", In: Nature inspired cooperative strategies for optimization (NICSO 2010), ed: Springer, pp. 65-74, 2010.

[25] G. Dhiman and V. Kumar, "Emperor Penguin Optimizer: A Bio-Inspired Algorithm for Engineering Problems", Knowledge-Based Systems, Vol. 159, pp. 20-50, 2018.

[26] G. Dhiman and V. Kumar, "Spotted Hyena Optimizer: A Novel Bio-Inspired Based Metaheuristic Technique for Engineering Applications", Advances in Engineering Software, Vol. 114, pp. 48-70, 2017.

[27] S. Mirjalili, S. M. Mirjalili, and A. Lewis, "Grey Wolf Optimizer", Advances in engineering software, Vol. 69, pp. 46-61, 2014.

[28] A. H. Gandomi, X.-S. Yang, and A. H. Alavi, "Cuckoo Search Algorithm: A Metaheuristic Approach to Solve Structural Optimization Problems", Engineering with Computers, Vol. 29, pp. 17-35, 2013.

[29] M. Dehghani, M. Mardaneh, and O. P. Malik, "FOA: 'Following' Optimization Algorithm for Solving Power Engineering Optimization Problems", Journal of Operation and Automation in Power Engineering, Vol. 8, No. 1, pp. 57-64, 2020.

[30] G. Dhiman, M. Garg, A. Nagar, V. Kumar, and M. Dehghani, "A Novel Algorithm for Global Optimization: Rat Swarm Optimizer", Journal of Ambient Intelligence and Humanized Computing, 2020.

[31] M. Dehghani, Z. Montazeri, A. Dehghani, and O. P. Malik, "GO: Group Optimization", Gazi University Journal of Science, Vol. 33, 2020.

[32] S. Saremi, S. Mirjalili, and A. Lewis, "Grasshopper Optimisation Algorithm: Theory and Application", Advances in Engineering Software, Vol. 105, pp. 30-47, 2017.

[33] M. Dehghani, Z. Montazeri, O. P. Malik, A. Ehsanifar, and A. Dehghani, "OSA: Orientation Search Algorithm", International Journal of Industrial Electronics, Control and Optimization, Vol. 2, No. 2, pp. 99-112, 2019.

[34] M. Dehghani, Z. Montazeri, and O. P. MALIK, "DGO: Dice Game Optimizer", Gazi University Journal of Science, Vol. 32, pp. 871-882, 2019.

[35] M. Dehghani, Z. Montazeri, O. P. Malik, H. Givi, and J. M. Guerrero, "Shell Game Optimization: A Novel Game-Based Algorithm", International Journal of Intelligent Engineering and Systems, Vol. 13, No. 3, pp. 246-255, 2020.

[36] M. Dehghani, Z. Montazeri, S. Saremi, A. Dehghani, O. P. Malik, K. Al-Haddad, J. M. Guerrero, "HOGO: Hide Objects Game Optimization", International Journal of Intelligent Engineering and Systems, Vol. 13, No. 4, pp. 216-225, 2020.

[37] M. Dehghani, Z. Montazeri, H. Givi, J. M. Guerrero, and G. Dhiman "Darts Game Optimizer: A New Optimization Technique Based on Darts Game", International Journal of Intelligent Engineering and Systems, Vol. 13, No. 5, pp. 286-294, 2020.

[38] N. E. Karkalos, A. P. Markopoulos, and J. P. Davim, "Evolutionary-Based Methods", In: Computational Methods for Application in Industry 4.0, ed: Springer, pp. 11-31, 2019.

[39] R. Storn and K. Price, "Differential Evolution-A Simple and Efficient Adaptive Scheme for Global Optimization over Continuous Spaces [r]", Berkeley: ICSI, 1995.

[40] K.-S. Tang, K.-F. Man, S. Kwong, and Q. He, "Genetic Algorithms and Their Applications", IEEE Signal Processing Magazine, Vol. 13, pp. 22-37, 1996.

[41] J. R. Koza, "Genetic Programming: A Paradigm for Genetically Breeding Populations of Computer Programs to Solve Problems", Stanford University, Department of Computer Science, 1990.

[42] H.-G. Beyer and H.-P. Schwefel, "Evolution Strategies-A Comprehensive Introduction", Natural Computing, Vol. 1, pp. 3-52, 2002.

[43] S. Mirjalili, "Biogeography-Based Optimisation", In: Evolutionary Algorithms and Neural Networks, ed: Springer, pp. 57-72, 2019.

[44] M. Dehghani, Z. Montazeri, O. P. Malik, G. Dhiman, and V. Kumar, "BOSA: Binary Orientation Search Algorithm", International Journal of Innovative Technology and Exploring 
Received: June 29, 2020. Revised: August 3, 2020.

Engineering (IJITEE), Vol. 9, pp. 5306-5310, 2019. 